\title{
EFECTO DE LA TÉCNICA DE ACODO EN Dracaena marginata, SOBRE LA CALIDAD DE LAS RAÍCES ${ }^{1}$
}

\author{
Vanessa Villalobos-Ramos ${ }^{2}$, María Isabel González-Lutz ${ }^{3}$, Alfredo Durán-Quirós ${ }^{4}$, Dennis Mora-Acedo ${ }^{4}$
}

\section{RESUMEN}

Efecto de la técnica de acodo en Dracaena marginata, sobre la calidad de las raíces. Se comparó el efecto del tiempo transcurrido desde la dilución de la hormona AIB y la forma de conservación de la hormona de enraizamiento AIB, y su relación con la forma de trabajo de cuatro acodadores (operarios que realizan los acodos) sobre la exportabilidad y la calidad de las raíces de acodos en cañas de Dracaena marginata de 4" y 6". Se utilizó un diseño irrestricto aleatorio con un arreglo factorial $4 \times 3$, y como unidad experimental cuatro minutos de trabajo. Se evaluaron tres variables en cada acodo: exportabilidad, ausencia de doble fila de raíces y largo de éstas, para lo cual se utilizaron modelos de regresión logística. Se encontraron interacciones entre el trabajo del acodador y el efecto de envejecimiento de la hormona para las tres variables modeladas, las cuales indican que uniformar el procedimiento con el que se realiza el acodo puede llevar a una mayor calidad y a aumentar la probabilidad de que cada caña sea exportable.

Palabras clave: Control de operaciones, exportación, costos de producción, ácido indolbutírico, reguladores de crecimiento.

\begin{abstract}
Effect of airlayering technique of Dracaena marginata on root quality. Effects of age and conservation of the IBA hormone and their relation to procedures of performing airlayers on quality of 4" and 6" Dracaena marginata roots were evaluated using a 4x3 factorial design. The factors were human labor and conservation of hormone. Logistic regression was used to predict probability of long roots, probability of single circumference of roots and exportability. Interactions between the effect of human labor and the effect of hormone levels on these three responses, can increase the cost up to $240 \%$ and suggest quality and exportability can increase if procedures are standardized.
\end{abstract}

Key words: Research of operations, export, production cost, indolbutyric acid, growth regulators.

\section{INTRODUCCIÓN}

Costa Rica es uno de los principales proveedores de plantas ornamentales a nivel mundial, tanto al mercado de Estados Unidos, como a la Comunidad
Económica Europea. En el año 2003, el 25,5\% del total de las plantas ornamentales que importó Europa, provenían de Costa Rica, lo que denota la importancia de esta actividad (CBI 2005).

1 Recibido: 23 de octubre, 2006. Aceptado: 26 de agosto, 2008. Investigación de Operaciones de Agricultura, Curso de Diseños Experimentales de la Maestría en Estadística. Universidad de Costa Rica, San José, Costa Rica.

2 Escuela de Economía Agrícola y Agronegocios, Facultad de Ciencias Agroalimentarias, Universidad de Costa Rica. vanevilla@gmail.com

3 Escuela de Estadística, Facultad de Ciencias Económicas, Universidad de Costa Rica. mariaisabel.gonzalezlutz@ucr.ac.cr

4 Estación Experimental Fabio Baudrit, Facultad de Ciencias Agroalimentarias, Universidad de Costa Rica. jose.duran@ucr.ac.cr; dennis. mora@ucr.ac.cr 
El cultivo de Dracaena marginata (conocida popularmente como marginata) es uno de los principales productos del rubro de ornamentales exportados por el país, dado que es uno de los cultivos con mayor área de siembra.

En Costa Rica no existen zonas de cultivo definidas. Se siembra en gran variedad de condiciones climatológicas que van desde pocos metros sobre el nivel del mar, hasta $1.200 \mathrm{msnm}$, en las zonas altas del Valle Central (Acuña et al. 1992).

La marginata es una planta de la familia Agavaceae, originaria de la isla de Madagascar, razón por la cual también es llamada "Madagascar dragon tree" (Osborne et al. 1984). Esta planta puede crecer hasta más de $4 \mathrm{~m}$ de alto, posee un tronco grueso, y generalmente varios tallos flexibles y largos, llamados "cañas" (Bernecker 2003). El cultivar verde oscuro es la planta más vendida, pero existen otros cultivares de diversos colores. La mayoría del material utilizado por los productores de Florida (USA), proviene del Caribe y de Centro América (Osborne et al. 1984).

La planta para exportación debe cumplir ciertos requerimientos específicos, que definen las actividades de producción, ya que se exportan cañas con dos o tres brotes o hijos terminales en el extremo superior, sin ningún otro tipo de crecimiento, ni follaje, en el resto de la caña y con una raíz bien desarrollada en el extremo inferior (Acuña et al. 1992). La raíz es producto de un acodo que se hace en la parte intermedia de una caña en la planta madre. La realización del acodo se realiza de cinco a siete semanas antes de cosechar la caña para exportación, dependiendo del tamaño de la planta a producir.

En la zona de Sarapiquí, provincia de Heredia, Costa Rica, en la elaboración del acodo se utiliza un alicate, con el cual se realiza un anillo en toda la circunferencia del tallo, eliminando el tejido de la corteza, a una altura que varía en función del tamaño de caña que se exportará y cuyos largos más frecuentes son: $4,6,12,18,24,36$ y 48 pulgadas de tallo o caña, considerando desde donde se inicia el acodo hasta donde salen los brotes apicales 5 .

\footnotetext{
5 García A. 2004. Metodología para realizar un acodo en $D$ marginata. Finca Los Gavilanes, Exportadora de Follajes y Flores S. A. Sarapiquí, Heredia. Comunicación personal.
}

En el acodo, los productores utilizan la hormona Ácido Indolbutírico (AIB), para inducir el crecimiento de las raíces, en una concentración acorde con el tamaño de planta a producir; entre más grande sea la planta, mayor concentración de hormona se requiere (Acuña et al. 1992). El AIB acelera el surgimiento de las raíces (Gaspar et al. 1996), aunque también uniformiza el tiempo de raiceo y produce una mejor calidad de raíces en cuanto a número, distribución y tamaño (Acuña et al. 1992).

Con frecuencia, los productores de D. marginata preparan grandes cantidades de hormona que al terminar el día aún no se ha gastado, por lo que es costumbre guardarla en una botella oscura para protegerla de la luz del sol y utilizarla al día siguiente sin el empleo de ningún sistema especial de conservación ${ }^{6}$. No se ha determinado si esta hormona que está envejecida, tendrá el mismo efecto que la hormona preparada al momento de ser aplicada, sin embargo, Wareing y Phillips (1985), indican que la hormona se degrada con la luz solar.

Es usual que las investigaciones de campo se enfoquen en la evaluación de los efectos de técnicas específicas de producción como por ejemplo, uso de fertilizante, plaguicidas u hormonas, asumiendo que las labores de los operarios están estandarizadas y por ende no van a modificar el efecto estudiado. Este supuesto no siempre se cumple y la no estandarización del trabajo de los operarios puede invalidar los resultados de esa evaluación.

El objetivo de este experimento fue evaluar la forma de realizar el proceso de acodado, sobre la calidad de los acodos de D. marginata, así como evaluar el impacto de estas diferencias sobre el costo de producción, en el caso de que se presenten.

\section{MATERIALES Y MÉTODOS}

\section{Diseño de campo}

El experimento se ubicó en una empresa exportadora de plantas de D. marginata, localizada en la zona

\footnotetext{
${ }^{6}$ Heinrich J. 2004. Manejo de la hormona AIB en las empresas productoras de D. marginata. Exportadora de Follajes y Flores S. A. Sarapiquí, Heredia. Comunicación personal.
}

AGRONOMÍA MESOAMERICANA 19(2): 209-219. 2009 
de San Ramón, en la Virgen de Sarapiquí, provincia de Heredia, durante los meses de setiembre a noviembre del 2004. Se utilizó una plantación de más de 10 años de edad, que se dedica a la producción de tamaños pequeños de caña (4 y 6 pulgadas). Las características del suelo donde se ubicó el experimento se muestran en el Cuadro 1. La distribución de la lluvia y temperaturas que prevalecieron durante el periodo experimental se muestran en las Figuras 1 y 2, respectivamente.

Se evaluó el efecto el tiempo transcurido desde su dilución hasta su aplicación, sobre la calidad de la raíz que produce el acodo. Las condiciones de manejo fueron: 1) a 15 horas de haberse preparado la hormona a temperatura ambiente y 2) dentro de un recipiente con hielo. Una tercera preparación fue elaborada previo a su aplicación.

Como segundo factor se evaluó el efecto de cada uno de los cuatro operarios que realizaron los acodos, en las características finales del acodo y en el porcentaje de cañas exportables. Para esto se escogieron cuatro personas que normalmente realizan la labor de acodar en la empresa y se evaluó el efecto de su manera particular de realizar el procedimiento de acodar, sobre la cantidad y calidad de acodos exportables.

Para la elaboración del acodo se utiliza un alicate, con el cual se realiza un anillo en toda la circunferencia del tallo, eliminando el tejido de la corteza, a una altura que varía en función del tamaño de caña a exportar. Con una brocha de una pulgada de grueso, se pone, sobre el corte, la hormona Ácido Indol Butírico (AIB), para inducir el crecimiento de las raíces. Luego se cubre el acodo con un trozo rectangular de papel aluminio de $10 \mathrm{~cm} \mathrm{X} 7 \mathrm{~cm}$. Estos tres pasos se realizan en un mismo día y momento de trabajo.

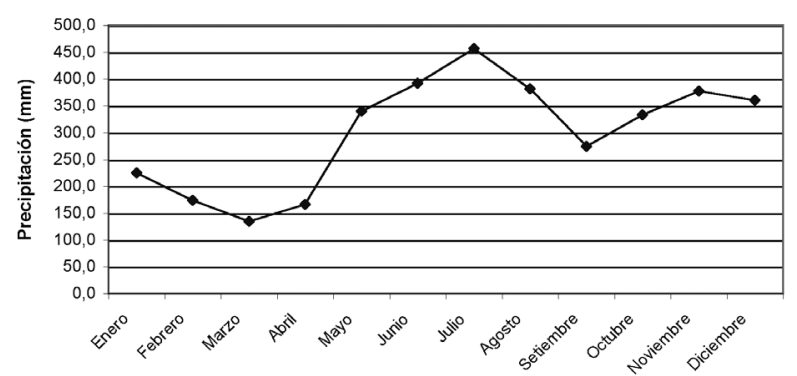

Figura 1. Distribución de la lluvia a lo largo del año en Puerto Viejo de Sarapiquí, Heredia, Costa Rica. Promedios mensuales (1975-2004).

Fuente: Instituto Meteorológico Nacional, Estación Puerto Viejo de Sarapiquí, latitud $10^{\circ} 28^{\prime}$; longitud $84^{\circ} 01^{\prime}$; altitud 37 msnm.

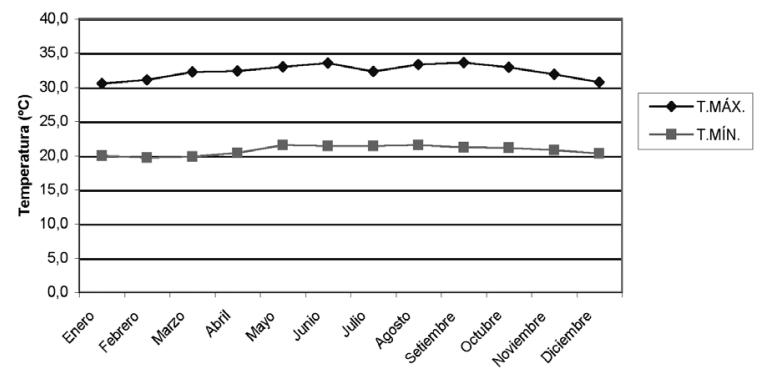

Figura 2. Temperaturas máxima y mínima en el cantón de Sarapiquí, Costa Rica. Promedios mensuales (1975-2004).

Fuente: Instituto Meteorológico Nacional, Estación Puerto Viejo de Sarapiquí, latitud $10^{\circ} 28^{\prime}$; longitud $84^{\circ} 01^{\prime}$; altitud 37 msnm.

Cuatro semanas después de haber realizado el acodo, cuando ya se observa el inicio de la formación

Cuadro 1. Características químicas del suelo de la finca en donde se evaluó la calidad de los acodos de Dracaena marginata, por efecto del proceso de acodar y envejecimiento de la hormona AIB. Sarapiquí, Heredia, Costa Rica. Abril, 2004.

\begin{tabular}{|c|c|c|c|c|c|c|c|c|c|c|c|c|c|}
\hline \multirow[b]{2}{*}{ Identificación } & \multirow{2}{*}{$\frac{\mathrm{H}_{2} \mathrm{O}}{\mathrm{pH}}$} & \multicolumn{4}{|c|}{$\operatorname{cmol}(+) / 1$} & \multicolumn{5}{|c|}{ mg/l } & \multicolumn{3}{|c|}{ Valor de Fertilidad } \\
\hline & & $\mathbf{K}$ & $\mathrm{Ca}$ & $\mathrm{Mg}$ & $\begin{array}{c}\text { Acidez } \\
\text { Ext. }\end{array}$ & $\mathbf{P}$ & $\mathrm{Fe}$ & $\mathrm{Cu}$ & $\mathbf{Z n}$ & Mn & $\begin{array}{l}\text { Sat. de } \\
\text { Acidez }\end{array}$ & $\begin{array}{l}\text { Suma de } \\
\text { Cationes }\end{array}$ & CICE \\
\hline Finca San Ramón & 5,0 & 0,41 & 6,12 & 0,77 & 0,37 & 10 & 141 & 11 & 3,2 & 27 & 4,82 & 7,30 & 7,67 \\
\hline
\end{tabular}


de las raíces, se procede a quitar el papel aluminio y a cubrir el acodo con un plástico de color negro o negro-plata, con el fin de evitar que las raíces adquieran un color verde, así como para acelerar su desarrollo y evitar que las raíces se abran o separen del tallo, puesto que es necesario que éstas vayan rectas, muy juntas entre sí y en la misma dirección del tallo.

Aproximadamente a las seis semanas después de haber realizado el acodo, se cortan las cañas unos ocho centímetros debajo del lugar donde surgieron las raíces.

Para la preparación del AIB, se diluye una cantidad conocida del producto comercial al $97 \%$, de acuerdo con la concentración deseada, en un volumen conocido de alcohol y agua (Acuña et al. 1992) y se almacena en condiciones de refrigeración y oscuridad (colocándola en un recipiente de un color que bloquee el paso de la luz).

La hormona AIB se consigue comercialmente en una concentración del 97\%. Para el experimento se utilizó una concentración de 2.000 ppm, que es la empleada por la empresa para los tamaños de caña a evaluar, por lo tanto para preparar un litro de hormona se pesó 2,06 gramos de IBA y se disolvió en 400 cc de alcohol al 95\% y se aforó con agua destilada hasta completar los 1.000 cc de solución de hormona.

Los tiempos que transcurrieron desde la dilución de la hormona y condiciones en que se mantuvo antes de su aplicación, se identificaron de la siguiente manera:

H1: Hormona diluida 15 horas antes de su aplicación y mantenida a temperatura ambiente.

$\mathrm{H} 2$ : Hormona diluida 15 horas antes de su aplicación, pero mantenida con hielo.

H3: Hormona diluida inmediatamente antes de su aplicación.

Los trabajadores que realizaron la labor de acodar las cañas se identificaron de la siguiente manera:

A1 $\rightarrow$ Acodador 1
A2 $\rightarrow$ Acodador 2

A3 $\rightarrow$ Acodador 3

A4 $\rightarrow$ Acodador 4

Se utilizaron tres repeticiones para cada uno de los 12 tratamientos. La unidad experimental correspondió al área de la cama de siembra en la que se hicieron acodos por un período de tiempo de trabajo de cuatro minutos; por lo tanto se requirió de 36 unidades experimentales. Se delimitó la unidad experimental en función del tiempo, dado que se requería comparar el efecto del trabajo de los acodadores.

Se seleccionó un lote de dieciocho hileras, con 50 metros de largo cada una y con una separación de un metro entre ellas. Se utilizó cada extremo de hilera para iniciar un tratamiento, de manera que en cada una se realizó dos tratamientos, uno en cada extremo.

Se seleccionó al azar el orden de aplicación de los tratamientos a cada hilera; en cada una se marcó hasta donde llegó cada trabajador en los cuatro minutos de trabajo. Evaluaciones preliminares permitieron establecer que un trabajador acoda cerca de seis cañas por minuto, lo que da un aproximado de veinticuatro cañas en cuatro minutos, por lo que se logró trabajar sin inconvenientes dos tratamientos en una misma hilera.

El diseño experimental utilizado fue un irrestricto aleatorio con un arreglo factorial de dos factores: cuatro acodadores y tres condiciones de manejo de la hormona (Montgomery 1991).

\section{Variables por evaluar}

La exportabilidad de las cañas se da cuando se cumplen ciertas condiciones en el largo, el grosor, la circunferencia del tallo con raíces, la posición de las mismas y su color. En este estudio, se evaluó el largo y grosor de la raíz, la presencia de doble fila de raíces y el porcentaje de la circunferencia del acodo con raíz, todas determinadas según una escala visual (Figura 3), en cada una de las cañas cosechadas de tamaño 4" y 6". La escala utilizada, propuesta por Omodeo 


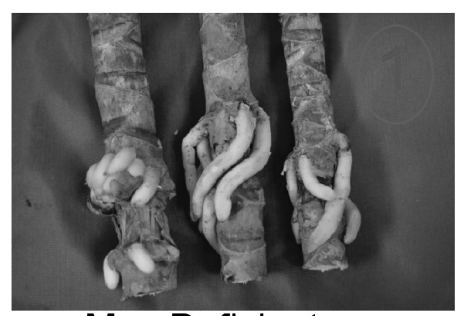

Muy Deficiente

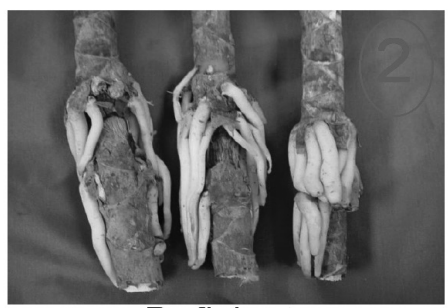

Deficiente

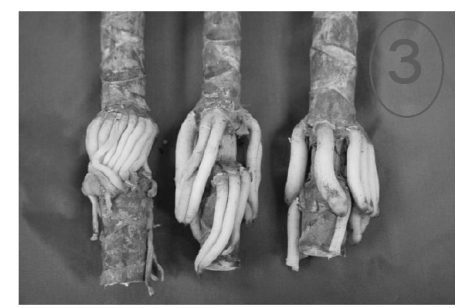

Bueno

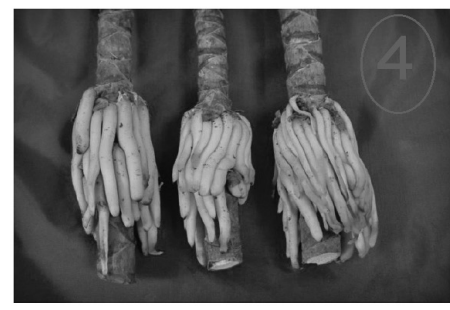

Sobreraiceado

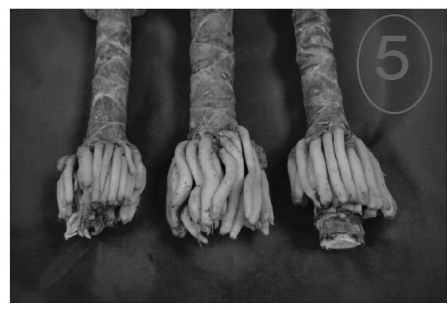

Óptimo

Figura 3. Clave para la evaluación de la calidad de la raíz de D. marginata. Omodeo et al. 2004.

et al. (2004, en prensa), se basa en cinco grados, a saber: muy deficiente, deficiente, bueno, sobreraizado y óptimo.

Se esperaba lograr calificar cada raíz como exportable según estas variables, sin embargo, producto de una omisión, no se realizó la envoltura de los acodos en plástico negro plata, labor que se debía hacer a las cuatro semanas de realizado el acodo; esto afectó el color, el crecimiento y la apariencia general de las raíces del mismo, por lo que se decidió sólo utilizar las variables grosor y porcentaje de circunferencia con raíces para calificar como exportable una caña.

Dado que la omisión en el emplasticado de los acodos se produjo en la totalidad del ensayo, se considera que no afectó los resultados obtenidos, por cuanto todos los tratamientos quedaron en igualdad de condiciones.

Se definió como aceptables aquellos acodos con raíces delgadas y con un $75 \%$ o más de la circunferencia del tallo con raíces, condiciones con las cuales se construyó una variable compuesta, denominada "exportable". Cada una de las variables tomó el valor de "1" cuando cumplió con la condición de aceptable para exportación, y de "0" cuando no. Los indicadores y los códigos utilizados se detallan en el Cuadro 2. En resumen, se analizaron tres variables: largo de la raíz, doble fila de raíces y exportabilidad.

En cada unidad experimental se sumaron los resultados obtenidos para las mediciones de cada caña y se calculó el porcentaje de cañas con las condiciones adecuadas para la exportación.

\section{Trabajo de campo}

En el momento de preparar los acodos, se trabajó con un observador para cada acodador, que contó la cantidad de acodos realizados, marcó en la cama de siembra hasta donde llegó el operario en el lapso de los cuatro minutos y tomó nota sobre la técnica con que se realizó el trabajo. Una persona con un cronómetro dio una señal de inicio y de conclusión para cada repetición de los tratamientos. 
Cuadro 2. Variables evaluadas en Dracaena marginata en cada unidad experimental, para determinar si las cañas eran exportables. Sarapiquí, Heredia, Costa Rica. Noviembre, 2004.

\begin{tabular}{|c|c|c|c|c|}
\hline \multirow[t]{2}{*}{ Variable } & \multicolumn{2}{|c|}{ Para cada caña } & \multicolumn{2}{|c|}{ Para cada unidad experimental } \\
\hline & Indicador & Código & Cálculo & Indicador \\
\hline \multirow[t]{2}{*}{ Largo } & \multirow[t]{2}{*}{$\begin{array}{l}\text { La raíz más frecuente mide } \\
\text { más de una pulgada }\end{array}$} & $1=\mathrm{Sí}^{\prime}$ & \multirow[t]{2}{*}{ Sumatoria/total de cañas } & $\begin{array}{l}\text { Porcentaje de cañas con raíz } \\
\text { con largo mayor a } 1 "\end{array}$ \\
\hline & & $0=\mathrm{No}$ & & \\
\hline \multirow[t]{2}{*}{ Grosor } & \multirow[t]{2}{*}{$\begin{array}{l}\text { La raíz más frecuente es } \\
\text { delgada }\end{array}$} & 1= Sí & \multirow[t]{2}{*}{ Sumatoria/total de cañas } & $\begin{array}{l}\text { Porcentaje de cañas con raíz } \\
\text { delgadas }\end{array}$ \\
\hline & & $0=\mathrm{No}$ & & \\
\hline \multirow[t]{3}{*}{ Doble } & \multirow{3}{*}{$\begin{array}{l}\text { Hay doble fila separada de } \\
\text { raíces }\end{array}$} & $1=$ No & \multirow[t]{3}{*}{ Sumatoria/total de cañas } & \multirow{3}{*}{$\begin{array}{l}\text { Porcentaje de cañas con una } \\
\text { sola fila de raíces }\end{array}$} \\
\hline & & & & \\
\hline & & $0=$ Sí & & \\
\hline \multirow[t]{4}{*}{ Circunferencia } & \multirow{4}{*}{$\begin{array}{l}\text { Porcentaje de la circunfe- } \\
\text { rencia de la caña que tiene } \\
\text { raíces }\end{array}$} & $1=$ más del $95 \%$ & & Porcentaje \\
\hline & & $2=\operatorname{del} 75$ al $95 \%$ & & Porcentaje \\
\hline & & $3=\operatorname{del} 50$ al $75 \%$ & & Porcentaje \\
\hline & & $4=$ menos del $50 \%$ & & Porcentaje \\
\hline \multirow[t]{2}{*}{ Exportable } & \multirow{2}{*}{$\begin{array}{l}\text { La circunferencia tiene có- } \\
\text { digo } 1 \text { ó } 2 \text { y el grosor tiene } \\
\text { código } 1\end{array}$} & 1= Sí & \multirow[t]{2}{*}{ Sumatoria/total de cañas } & \multirow{2}{*}{$\begin{array}{l}\text { Porcentaje de cañas que son } \\
\text { exportables }\end{array}$} \\
\hline & & $0=\mathrm{No}$ & & \\
\hline
\end{tabular}

Algunas características de la técnica de acodado de cada trabajador que se determinaron fueron la cantidad de hormona usada, cantidad y estilo de los movimientos para realizar el acodo y posición donde se colocó la hormona en el acodo.

Luego de seis semanas de realizado el acodo, se efectuó la cosecha, la cual se hizo toda el mismo día. Se cosecharon 1.047 cañas.

\section{Metodología de análisis}

Dado que las variables a medir en este caso son dicotómicas (sólo pueden asumir dos categorías: éxito o fracaso), se decidió utilizar modelos de regresión logística para el análisis estadístico (Johnson 2000) donde la variable respuesta (dependiente) es la probabilidad de obtener la condición de calidad esperada en el acodo en función de los tres factores a probar (variables independientes) descritos en el apartado Diseño de Campo. Este método de análisis permite determinar si un efecto (acodador, hormona o las combinaciones de ambos) modifica de manera significativa la probabilidad de obtener las condiciones aceptables de calidad para los acodos.

En este modelo de regresión la interpretación se hace con base en la significancia de las razones de ventaja para lograr acodos exportables asociadas a cada uno de los niveles de los factores, de manera que se determina cuál es la ventaja de alguno de los métodos de manipulación de la hormona o de alguno de los acodadores o combinaciones de ambos en particular, sobre uno que se fija como base de comparación.

Se ajustaron tres modelos de regresión logística, uno para cada variable respuesta: la proporción de acodos con largo igual o mayor a una pulgada (largo=1), la proporción de acodos sin doble fila de raíz (doble=1) 
y la proporción de acodos exportables (exportable $=1$ ). Para cada una de ellas, el nivel de referencia con el que se comparó, en el caso del factor acodador, fue el acodador cuatro y en el caso del factor hormona, fue la hormona vieja (con más de 15 horas de preparación y mantenida a temperatura ambiente) y en el caso de las combinaciones de ambos, fue contra el acodador cuatro utilizando hormona vieja.

Se utilizó un nivel de significancia de $10 \%$ porque este es un estudio cuyo fin primordial es obtener información de base para futuras investigaciones. Los datos se procesaron con el paquete estadístico SAS.

\section{RESULTADOS}

\section{Caracterización de las variables respuesta}

La proporción de acodos con la característica deseada para cada una de las variables evaluadas se muestra en el Cuadro 3.

De manera general se tuvo acodos con bastantes raíces, aunque tupidas y cubriendo la mayor parte de la circunferencia del acodo, inclusive con un alto porcentaje de doble fila de raíces. Probablemente la alta incidencia de raíces haya provocado que la mayoría fueran delgadas.

También se consideró que el hecho de que los acodadores estaban siendo observados constantemente por las personas que controlaban el tiempo y la técnica

Cuadro 3. Proporción de acodos de Dracaena marginata con características apropiadas para exportación. Sarapiquí, Heredia, Costa Rica. Noviembre, 2004.

\begin{tabular}{lc}
\hline Variable & Proporción (\%) \\
\hline Circunferencia de raíces en el acodo & 90,34 \\
(con el 75\% o más) & \\
Largo de raíces del acodo (1” o más) & 17,11 \\
Una sola fila de raíces & 47,80 \\
Raíces delgadas & 82,12 \\
Acodos exportables & 73,42 \\
\hline
\end{tabular}

de trabajo, pudo haber incidido en la gran cantidad de acodos con doble fila de raíz, ésto les pudo haber creado una sensación de vigilancia que pudo provocar aplicaciones excesivas de hormona, produciendo una alta proporción de acodos dobles $(52,20 \%)$, que si bien no impide la exportación de la caña, si le disminuye su calidad, pues el mercado prefiere una sola fila de raíces bien desarrolladas.

En lo referente a la circunferencia del acodo que estaba cubierta con raíces, se observa en los resultados que un 90,34\% de los acodos estaba en condiciones de ser exportado, ya que tenían un $75 \%$ o más de la circunferencia del acodo con raíces.

En su mayoría $(82,89 \%)$ las raíces desarrollaron con menos de una pulgada de largo, lo cual está relacionado con la falta de cobertura plástica de los acodos. Este resultado refuerza la importancia de cubrir el acodo con el plástico negro-plata, aspecto que aunque no estaba bajo evaluación, sí quedó demostrado con la situación que se presentó.

El 82,12\% de los acodos presentaron raíces delgadas, esto es favorable para efectos del mercado, ya que es una condición solicitada por los compradores.

Finalmente, para la variable que resume la condición de exportabilidad, según la cobertura de la circunferencia del tallo con raíz y su grosor, se encontró que un $73,42 \%$ de los acodos fueron exportables, lo cual es un porcentaje aceptable.

Como valores de referencia para catalogar la eficiencia del trabajo de los acodadores, se estableció la relación de acodos realizados por minuto para cada uno de ellos (Cuadro 4). Se dieron valores desde 8,55 acodos por minuto hasta 5,97 .

\section{Resultados de los modelos de regresión}

Las razones de ventaja obtenidas para cada uno de los niveles de los efectos correspondientes y su respectiva probabilidad asociada, se presentan en el Cuadro 5. Las razones de ventaja se deben interpretar como favorables (mayor posibilidad de obtener la calidad deseada) en el caso de que sean mayores al valor de uno 
Cuadro 4. Acodos totales y por minuto de Dracaena marginata realizados por cada operario. Sarapiquí, Heredia, Costa Rica. Noviembre, 2004.

\begin{tabular}{ccc}
\hline Acodador & $\begin{array}{c}\text { Total acodos } \\
\text { realizados }\end{array}$ & Acodos/minuto \\
\hline 1 & 252 & 6,63 \\
2 & 227 & 5,97 \\
3 & 325 & 8,55 \\
4 & 243 & 6,39 \\
\hline
\end{tabular}

y como desfavorables en el caso de que sean menores a uno. Serán favorables o desfavorables en relación con el nivel con el que se comparan en cada caso.

Como puede observarse, hay efectos de interacción significativos en las tres variables analizadas. Esto implica que el efecto del acodador y el de la hormona no son independientes, sino que se dio una interación acodador-hormona.
Si el estudio se hubiera realizado solamente con el objetivo de evaluar el efecto del método de conservación de la hormona después de diluida, se habría llegado a la conclusión de que usar hormona fresca (H3) está en una desventaja de 0,7 a 1 con respecto al uso de hormona del tratamiento $\mathrm{H} 1$ para la obtención de acodos con doble fila de raíz, mientras que usar hormona conservada con hielo tuvo una ventaja de 1,3 a 1 . Se analizó este resultado a la luz del método empleado por el acodador, se puede advertir que la relación no es la misma para todos ellos: con el método del acodador dos, la hormona fresca tiene ventaja (3,1 a 1) respecto a la hormona del tratamiento $\mathrm{H} 1$, si ésta es utilizada por el acodador cuatro, pero con el método del acodador tres tiene desventaja (0,3 a 1). Sin embargo, la hormona conservada con hielo (tratamiento $\mathrm{H} 2$ ), independientemente de quién la aplicó, no hace diferencia alguna con la hormona del tratamiento $\mathrm{H} 1$, aplicada por el acodador cuatro. Es evidente que no es posible llegar a una única conclusión para cada factor, puesto que ambos factores no son independientes.

Cuadro 5. Razones de ventaja y significancias de los efectos para la predicción de las probabilidades de éxito en las variables "largo" "doble" y "exportable" de Dracaena marginata. Sarapiquí, Heredia, Costa Rica. Noviembre, 2004.

\begin{tabular}{lcccccc}
\hline Efectos & \multicolumn{2}{c}{ Largo } & \multicolumn{2}{c}{ Sin doble } & \multicolumn{2}{c}{ Exportable } \\
\hline & $\begin{array}{c}\text { Razón de } \\
\text { ventaja }\end{array}$ & $\begin{array}{c}\text { Significan- } \\
\text { cia Wald }^{\text {a }}\end{array}$ & $\begin{array}{c}\text { Razón de } \\
\text { ventaja }\end{array}$ & $\begin{array}{c}\text { Significan- } \\
\text { cia Wald }\end{array}$ & $\begin{array}{c}\text { Razón de } \\
\text { ventaja }\end{array}$ & $\begin{array}{c}\text { Significan- } \\
\text { cia Wald* }\end{array}$ \\
\hline Acodador 1 (A1) & 1,2 & 0,297 & 0,6 & 0,000 & 1,6 & 0,001 \\
Acodador 2 (A2) & 0,8 & 0,148 & 3,1 & 0,000 & 0,5 & 0,000 \\
Acodador 3 (A3) & 1,2 & 0,273 & 0,9 & 0,292 & 0,6 & 0,000 \\
Hormona fresca (H3) & 1,2 & 0,201 & 0,7 & 0,000 & 1,0 & 0,801 \\
Hormona hielo (H2) & 0,9 & 0,574 & 1,3 & 0,017 & 0,9 & 0,611 \\
A1* H3 & 1,3 & 0,227 & 1,2 & 0,227 & 1,4 & 0,154 \\
A1* H2 & 0,9 & 0,636 & 0,9 & 0,620 & 0,9 & 0,491 \\
A2* H3 & 0,9 & 0,653 & 3,1 & 0,000 & 1,3 & 0,152 \\
A2* H2 & 0,6 & 0,035 & 0,8 & 0,234 & 0,7 & 0,076 \\
A3* H3 & 1,3 & 0,166 & 0,3 & 0,000 & 0,5 & 0,000 \\
A3* H2 & 1,1 & 0,777 & 1,1 & 0,495 & 1,7 & 0,002 \\
\hline
\end{tabular}

* Probabilidades menores a 0,10 se consideran significativas. Son significativas las razones de ventaja que se presentan en negrita. 
En lo que se refiere a exportabilidad de las cañas, los resultados son similares a los que se dieron para la obtención de acodos dobles: la presencia de efectos combinados significativos invalida las conclusiones a las que se podría llegar para los efectos simples. Por ejemplo, los resultados de utilizar hormona fresca o bien hormona conservada con hielo (tratamiento H2), no presentaron diferencias significativas con la hormona del tratamiento $\mathrm{H} 1$ (las probabilidades asociadas son 0,801 y 0,611 para los efectos simples tratamiento $\mathrm{H} 2$ y tratamiento $\mathrm{H} 3$ respectivamente), sin embargo, la hormona del tratamiento $\mathrm{H} 1$ con hielo, si es aplicada por el acodador tres, tuvo una ventaja de 1,7 a 1 con respecto a la hormona de tratamiento $\mathrm{H} 1$ aplicada por al acodador cuatro, pero una desventaja de 0,7 a 1 cuando fue aplicada por el acodador dos.

Una razón de ventaja de 1,7 significa una probabilidad de exportar un $70 \%$ más de cañas. Sin embargo los resultados indican que este aumento del $70 \%$ en exportabilidad no se debe únicamente al efecto de cambiar el tiempo transcurrido luego de la dilución de la hormona o el método de conservación de la hormona, sino que interviene de manera significativa la técnica de trabajo del operario. Como ejemplo, en este caso se tiene que si se cambia del acodador tres al acodador dos, ambos con el mismo tipo de hormona, con el acodador dos se reduciría en un 50\% la cantidad de cañas exportables, mientras con el acodador tres se aumentaría en un $70 \%$ dicha cantidad, ambos comparados con el acodador cuatro usando hormona del tratamiento $\mathrm{H} 1$.

Si se calcula el costo de los efectos mencionados, asumiendo que un acodador gana $\phi 6,65$ por acodo realizado $^{7}$, independientemente de que éste llegue a ser exportable o no, obtenemos un costo de mano de obra por realizar 100 acodos de $\phi 665$, en el Cuadro 6 se muestran las diferencias de costos que se tendrían en función de los efectos de interacción que resultaron significativos.

El porcentaje de exportabilidad que obtuvo cada operario mostró diferencias económicas importantes,

7 Heinrich, J. 2004. Costos asociados a la confección de acodos en las fincas de marginata. Exportadora de Follajes y Flores S. A. Sarapiquí, Heredia, Costa Rica. Comunicación personal.
Cuadro 6. Costo de mano de obra requerido en acodar para obtener 100 cañas de D. marginata exportables. Sarapiquí, Heredia, Costa Rica. Noviembre, 2004.

\begin{tabular}{cccc}
\hline Acodador & Hormona & $\begin{array}{c}\text { Razón de } \\
\text { ventaja }\end{array}$ & $\begin{array}{c}\text { Costo por 100 aco- } \\
\text { dos exportables }(\boldsymbol{\ell})\end{array}$ \\
\hline A2 & Hielo & 0,7 & 950,00 \\
A3 & Fresca & 0,5 & $1.330,00$ \\
A3 & Hielo & 1,7 & 391,18 \\
A4 & Vieja & Referencia & 665,00 \\
\hline
\end{tabular}

sin considerar aún el costo de la caña al momento que se realiza el acodo, puesto que para entonces ya se ha invertido en ésta insumos y labores de mano de obra y se seguirá invirtiendo a pesar de que al final de la cadena de procesos ese acodo se vaya a desechar.

En el Cuadro 7 se presentan los resultados de las observaciones hechas a cada operario sobre su manera particular de realizar los acodos. Se incluyen observaciones sobre la cantidad de movimientos del alicate utilizados para hacer el acodo, la colocación de la hormona en el acodo, la cantidad de veces que se untó la hormona y la cantidad de hormona utilizada.

El operario uno tuvo el mayor sobrante de hormona (84cc), es decir, fue el que menos hormona gastó; también éste realizó dos movimientos cortos de la brocha sobre el área central del corte, uno a la derecha y otro a la izquierda completando la circunferencia, impregnando con hormona la brocha en cada movimiento y si bien no fue el más rápido en realizar el trabajo, ocupó la segunda posición en la razón de acodos por minuto teniendo un promedio de 6,63, aspecto que también demuestra la experiencia del acodador en la labor.

El operario tres por el contrario, fue el que utilizó mayor cantidad de hormona para realizar su trabajo, ya que no tuvo sobrante de hormona, sin embargo, también fue el más eficiente ya que tuvo el mayor porcentaje de acodos por minuto $(8,55)$ de modo que el mayor gasto de hormona está asociado con un mayor número de acodos realizados. El Cuadro 7 nos indica que este operario tiene una manera particular de realizar el acodo. 
Cuadro 7. Características observadas para cada operario al acodar Dracaena marginata. Sarapiquí, Heredia, Costa Rica. Noviembre, 2004.

\begin{tabular}{lcccc}
\hline \multirow{2}{*}{ Técnica de trabajo } & \multicolumn{5}{c}{ Acodador } \\
\cline { 2 - 5 } & $\mathbf{1}$ & $\mathbf{2}$ & $\mathbf{3}$ & $\mathbf{4}$ \\
\hline Hormona sobrante $(\mathrm{cc})^{*}$ & 84 & 64 & 0 & 30 \\
Acodos/minuto & 6,63 & 5,97 & 8,55 & 6,39 \\
Movimientos del Alicate & Dos cortos & Dos cortos & Uno solo completo & Dos cortos \\
Trazo de la brocha & Al centro del corte & Al centro del corte & Arriba del corte & Arriba del corte \\
Sumergir brocha en hormona & Dos veces & Una vez & Una vez & Dos veces \\
\hline
\end{tabular}

* A cada acodador se le entregó la misma cantidad de hormona.

\section{DISCUSIÓN}

El efecto del operario al efectuar el acodo mostró diferencias significativas en el presente experimento, tanto de manera simple como en interacción con el envejecimiento de la hormona para las variables acodo con raíces dobles y número de acodos exportables. Los resultados confirman que los operarios y específicamente sus técnicas de trabajo, inciden en la cantidad y calidad de los acodos de las cañas de marginata.

El efecto del tiempo de conservación de la hormona que se midió en tres niveles en este experimento, mostró diferencias en los resultados observados dependiendo del acodador que realizó el trabajo.

Ha sido frecuente en agricultura, que se lleve a cabo investigaciones para comparar diferentes insumos o dosis de los mismos, sin tomar en cuenta que los resultados que se obtengan también están relacionados con los trabajadores que llevan a cabo las tareas.

Los resultados muestran la necesidad de realizar estudios posteriores que permitan validar el uso de una técnica eficiente y estandarizada para la realización de los acodos en D. marginata.

Se pone en evidencia que en el campo de la investigación de operaciones en la agricultura, se debe poner especial atención a las técnicas de trabajo de los empleados del campo y al adiestramiento del personal, aunque se trate de labores que se han considerado como sencillas o básicas y hasta de bajo costo y que, sin embargo, por las consecuencias económicas demostradas, parece que no han sido consideradas con la importancia que realmente tienen.

En este ensayo, para una misma labor, como es la realización del proceso de acodado de las cañas de marginata, existieron marcadas diferencias no sólo en la eficiencia de trabajo y en la cantidad de acodos que un operario puede realizar, sino también en la efectividad de tales labores, ya que los resultados indican que los cuatro operarios evaluados en este ensayo, produjeron resultados diferentes en cuanto a cantidad de acodos exportables, así como también en cuanto a la eficiencia económica de su labor, por lo que dos operarios realizando el mismo trabajo, pueden generar costos de hasta cuatro veces más para obtener la misma cantidad de acodos exportables.

Es indispensable que las empresas que producen D. marginata realicen una evaluación de los métodos y procesos utilizados por los empleados de campo que realizan los acodos y que busquen estandarizar los procedimientos mediante capacitación, seleccionando como referencia, a aquellos operarios que obtengan los mejores resultados.

Con relación a la duda de si la hormona que tiene 15 horas de preparada pierde efecto, los resultados indican que al evaluar los efectos simples, no hubo 
diferencias significativas en cuanto al número de cañas exportadas, entre la hormona preparada quince horas antes y mantenida a temperatura ambiente, con respecto a la hormona diluida 15 horas antes y mantenida en frío y la hormona fresca, sin embargo, al evaluar los efectos combinados acodador-hormona, se obtuvo que existió ventaja para algunas formas de conservar la hormona, según sea el operario que las utilizó. Por lo tanto, más que la forma de conservar la hormona luego de diluida (a temperatura ambiente o en hielo), lo importante fue quien y cómo la utilizó.

\section{LITERATURA CITADA}

Acuña, B; Jiménez, A.C.; Franco, JA; Murillo, G; Ramírez J; Gamboa, J; Fernández A. 1992. Técnicas para la producción de Dracaena marginata en Costa Rica. EUNED, San José, Costa Rica, 88 p.

Bernecker, R. 2003. Dracaena marginata. Documento virtual. Consultado 20 julio 2005. Disponible en: http:// www.dracaena.com/Marginata.asp

Centre for the Promotion of Imports from developing countries (CBI). 2005. Research for the EDP Central
América young plant material, ornamental plants and foliage. Material mimeografiado.

Gaspar, T; Kevers, C; Penel, C; Greppin, H; Reid, D; Thorpe, T. 1996. Plant hormones and plant growth regulators in plant tissue culture. Vitro Cell Dev. BiolPlant. 32: 272-289.

Montgomery, DC. 1991. Diseño y análisis de experimentos. Grupo editorial Iberoamérica, México. 172 p.

Johnson, DE. 2000. Métodos multivariados aplicados al análisis de datos. International Thomson Editores, México. 240 p.

Omodeo, P; González, MI; Mora, D; Durán A. 2004. Efecto del Acido Indolbutirico (AIB) y el uso de papel alumnio sobre la calidad de los acodos de Dracaena marginata". Agricultura Tropical. Cosa Rica. Sin publicar.

Osborne, LS; Chase, AR; Henley, RW. 1984. Dracaena marginata. AREC-A Foliage Plant Research Note, University of Florida. 6 p.

Wareing, PF; Phillips, IDJ. 1985, Growth and differentiation in plants. 3 ed. Pergamon Press, Gran Bretaña. 343 p. 